\title{
Characterization of $\mathrm{BiVO}_{4}$ Powders and Thin Films by Electron Microscopy and Electron Energy Loss Spectroscopy
}

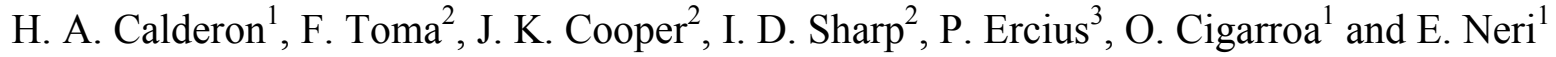 \\ 1. Depto. Física, Instituto Politécnico Nacional - ESFM, UPALM Zacatenco Ed. 9, CDMX, Mexico. \\ 2. Chemical Sciences Division and JCAP, LBNL, Berkeley CA, USA. \\ 3. Molecular Foundry-NCEM, LBNL, Berkeley CA, USA.
}

BiVO4 (BVO) is a promising material for photoelectro-chemical water splitting by using visible light. It is formed by abundant and nontoxic elements i.e. low cost and environmentally friendly. BVO has three polymorphs: orthorhombic, tetragonal, and monoclinic. The monoclinic phase (m-BVO) phase exhibits a much higher photocatalytical activity due to its favorable band gap $(2.4-2.5 \mathrm{eV})$ in the visible region of the electromagnetic spectrum, and a valence band position suitable for driving water oxidation under illumination. A previous investigation using scanning transmission electron microscopy (STEM) and energy loss spectroscopy (EELS) has shown a pronounced surface reduction of monoclinic BVO particles i.e., within a $5 \mathrm{~nm}$ thick shell, the oxidation state of $\mathrm{V}$ is reduced from +5 to about +4 [1]. This demands for around 15\% oxygen vacancies of charge neutrality is assumed.

This investigation deals with $\mathrm{H}_{2}$ loading of m-BVO powders and thin films in an effort to avoid having vacancies and thus promote a better conductivity and an improved performance of monoclinic scheelite. The synthesis procedure has been already published together with the roles of hydrogen impurity and oxygen vacancy defects on defining the conductivity, and hence photoelectrochemical (PEC) performance characteristics of $\mathrm{m}-\mathrm{BVO}$ by using a combination of experiment and theory [2]. Here a report is given on the characterization of $\mathrm{m}-\mathrm{BVO}$ powders and thin film by transmission electron microscopy and EELS. The TEAM 0.5 electron microscope has been used under an accelerating voltage of $80 \mathrm{KV}$ and imaging has been done in conditions of low dose both in STEM and TEM modes. The TEM images are used to reconstruct the exit wave and obtain phase images.

Figure 1 shows results of pure m-BVO powder particles and the corresponding EELS measurements. As expected there is an energy shift of the $\mathrm{V}-\mathrm{L}_{2,3}$ white lines of around $1 \mathrm{eV}$ as the measurement is done at the surface (1) or in the bulk (8). The insert gives the phase recovery as a function of distance in the phase image. It is a normal profile for a wedge sample. Figure 2 shows results typical for powder particles of $\mathrm{H}_{2}$ loaded $\mathrm{m}-\mathrm{BVO}$. In this case the phase image is slightly tilted from the [110] zone axis. There is a contrast variation near the surface that produces an increase in the phase recovery as the inset shows. Typical EELS spectra are given in Fig. 2b, the shift of the $\mathrm{V}-\mathrm{L}_{2,3}$ edge is now disappeared. Apparently the oxidation state of $\mathrm{V}$ becomes constant. The thin foils results are summarized in Figure 3. A phase image is shown after an EWR procedure with 40 images. Fig. $3 \mathrm{~b}$ shows EELS spectra in the region of interest with little to no shift as a result of the $\mathrm{H}_{2}$ loading. Loading $\mathrm{m}-\mathrm{VBO}$ with hydrogen appears to have a clear effect on the oxidation state of $\mathrm{V}$ near the surface of powders and thin film.

\section{References}

[1] M. D. Rossell et al, Chemistry of Materials 27 (2015), p. 3593.

[2] J. K. Cooper et al, Chemistry of Materials 28 (2016), p. 5761.

[3] HAC acknowledges support of CONACYT (FOINS 75 2012/75) and IPN (COFAA-SIP). Work at the Molecular Foundry is supported by the Office of Science, the Office of Basic Energy Sciences, the 
U.S. Department of Energy under Contract No. DE-AC02-05CH11231.

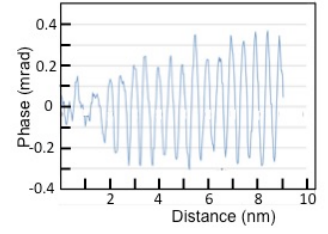

Fig. 1. Pure m-BVO powder. (a) Phase image after EWR procedure. (b) EELS spectra taken

perpendicularly to the surface at intervals of around $1.5 \mathrm{~nm}$.

Spectrum 1 is taken at the surface.

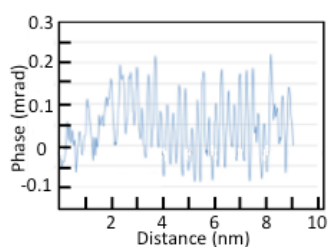

Figure 2. $\mathrm{H}_{2}$ loaded powder particle. (a) Phase image with a phase recovery inset. (b) EELS spectra taken along a profile perpendicular to the surface at intervals around $1.5 \mathrm{~nm}$.

Figure 3. $\mathrm{H}_{2}$ loaded m-BVO thin film. (a) Phase image. (b) EELS spectra taken along a profile perpendicular to the surface at intervals of around $1.5 \mathrm{~nm}$.
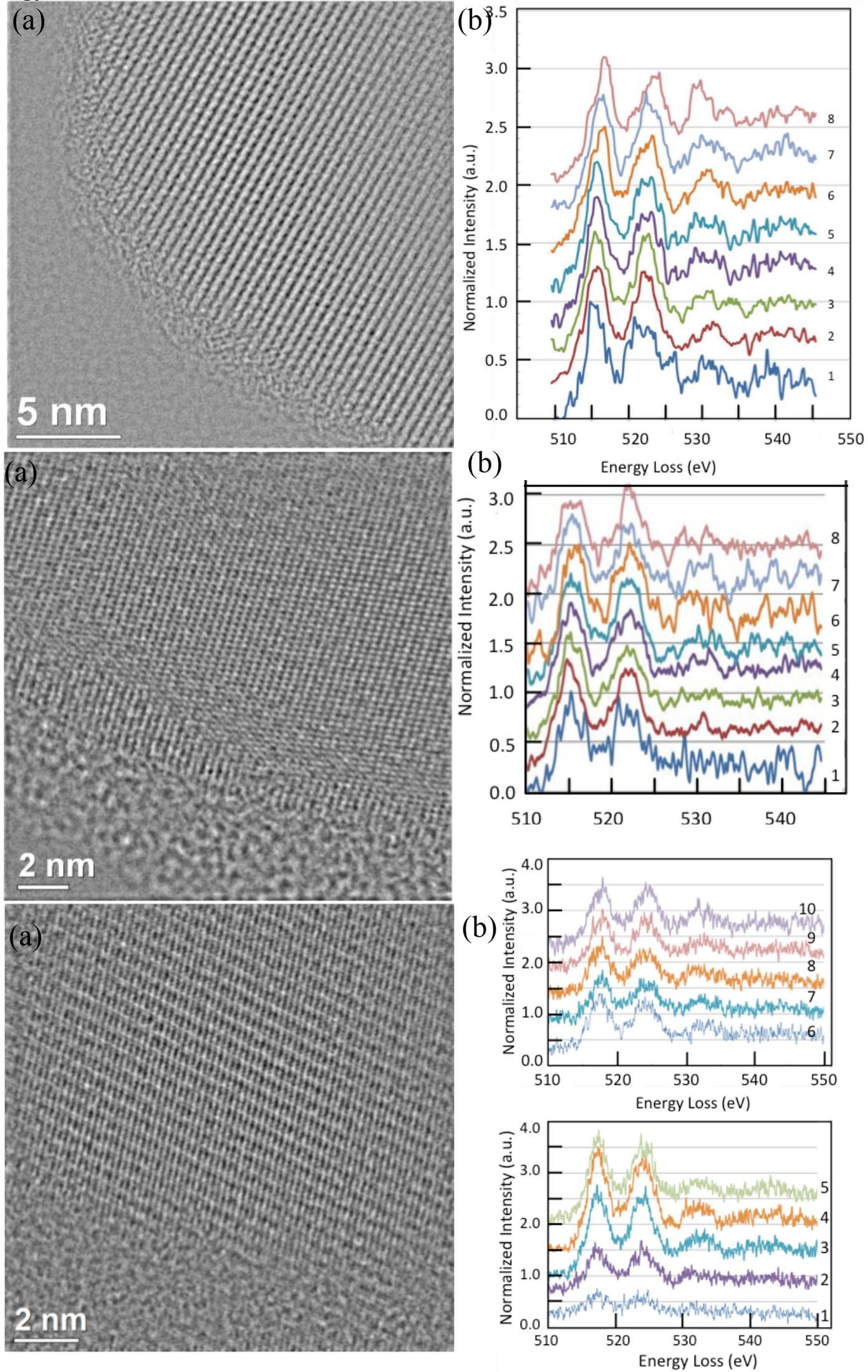